\title{
Phases Coexistence and Surface Tensions for the Potts Model
}

\author{
L. Laanait*, A. Messager, and J. Ruiz
}

Centre de Physique Théorique ${ }^{\star \star}$, F-13274 Marseille Cedex 2, France

\begin{abstract}
The $q$ states Potts model exhibits a first order phase transition at some inverse temperature $\beta_{t}$ between "ordered" and "disordered" phases for $q$ large as proved in [1]. In space dimension 2 we use the duality transformation as an internal symmetry of the partition function at $\beta_{t}$ to derive an estimate on the probability of a contour. This enables us to prove the preceding result and the following new results:

(i) The discontinuity of the mass gap at $\beta_{t}$.

(ii) The existence of a strictly positive surface tension between two ordered phases up to $\beta_{t}$.

(iii) The existence of a non-zero surface tension between an "ordered" and the "disordered" phase at $\beta_{t}$.
\end{abstract}

\section{Introduction}

An usual situation in statistical mechanics is the coexistence of several ordered phases (corresponding to the different ground states of the Hamiltonian). Such a situation is very well described by the Pirogov-Sinai theory [8]. At high temperature there is a unique phase (the "disordered one"). We sketch two typical behaviours.

1. There is a second order phase transition at $\beta_{c}$ (critical inverse temperature) and above $\beta_{c}$ a surface tension between different ordered phases which goes to zero at $\beta_{c}$. (This situation occurs in many models such as the Ising model $[3,4]$.)

Moreover at high temperature the two points function decays exponentially in the distance with a mass gap vanishing at $\beta_{c}$, as shown by Simon [2] (see also previous works of Dobrushin and Pechersky [28]).

2. The phase transition is first order at $\beta_{t}$. Such a situation was exhibited by Dobrushin and Shlosman [22] for continuous spin and by Kotecky and Shlosman

* On leave from Ecole Normale Supérieure Takaddoum, Rabat, Marocco

$\star \star$ Laboratoire propre du CNRS 
[1] for the Potts model. They use as an essential tool the reflexion positivity. We want to emphasize that this transition between ordered and disordered phases does not correspond to ground states of the Hamiltonian.

We exhibit an internal symmetry of the partition function for the two dimensional Potts model: the duality transformation (in fact this symmetry exists for a wide class of models). This symmetry sends the partition function with free boundary conditions (b.c.) at $\beta_{t}$ into the partition function with " 0 " b.c. at $\beta_{t}-$ up to boundary terms. It enables us to prove the Peierls condition [3] for the contours which separates the "ordered" and the "disordered" regions with arbitrary boundary conditions. From these basic estimates we get the following results:

a) The coexistence of $q$ ordered phases and of a disordered one at $\beta_{t}$. Moreover the spontaneous magnetization is discontinuous at $\beta_{t}$.

b) The discontinuity of the mass gap at $\beta_{t}$.

c) The existence of a strictly positive surface tension between two arbitrary ordered phases up to $\beta_{t}$.

d) The existence of a surface tension between an ordered phase and the disordered phase at $\beta_{t}$.

The paper is organized as follows:

- Chapter 2 fixes the notations and contains the results

- Chapter 3 describes the duality transformation.

- Chapter 4 concerns our basic estimate for the probability of a contour.

- Chapter 5 is devoted to the proof of the theorems.

\section{Definitions and Results}

The cell complex formalism is very efficient for the study of duality in statistical mechanics $[9,26,27]$ as noticed by Drouffe [25]. So we will introduce the Potts model with this formalism in order to have a coherent formulation: the lattice will be considered as a cell complex and the configuration as 0-chains taking their values in the group $\mathbb{Z} q$.

\section{II.1. Cell Complex Formalism}

We refer the reader to Alexandroff's book [17]. A cell complex is a set of elements called cells with the following properties: to each cell is assigned a non-negative integer called its dimension, the upper bound of the cell dimensions is called the dimension of the complex. Each cell of dimension $s_{r}$ is in correspondence with another cell $\left(-s_{r}\right)$ of the same dimension (cell with opposite orientation); to two cells $s_{r}$ and $s_{r-1}$ is assigned an integer $I\left(s_{r}, s_{r-1}\right)$ called the incidence number such that: $I\left(-s_{r}, s_{r-1}\right)=I\left(s_{r-1},-s_{r}\right)=-I\left(s_{r}, s_{r-1}\right)$. A cell complex is said to be an $a$-complex if the following condition is satisfied: for any two cells $s_{r+1}, s_{r-1}$

$$
\sum_{s_{r}} I\left(s_{r+1}, s_{r}\right) I\left(s_{r}, s_{r-1}\right)=0 .
$$

(Another terminology is sometimes used: a cell space instead of a cell complex, and a cell complex instead of $a$-complex; the above have been introduced by Alexandroff in [17].) 
An $r$-chain $c_{r}$ over the coefficient domain $G$ ( $G$ is a ring with unity) is an odd function of the $r$-cells over $G$. Any $r$-chain may be written as a sum of monomial chains (the group law of $G$ is denoted additively).

$$
c_{r}=\sum_{s_{r}} m_{s_{r}} s_{r} .
$$

The monomial chain $m \cdot s_{r}$ is the chain that takes the value $m$ on $s_{r}$ and vanishes on the $r$-cells different of $\pm s_{r}: m_{s_{r}}=c_{r}\left(s_{r}\right)$. The set of $r$-chains over $G$ forms an abelian group denoted $C^{r}(S, G)$. The $r$ chains of $C^{r}(S, \mathbb{Z})\left[\right.$ denoted $\left.C^{r}(S)\right]$ are called integral chains, the rank of this group is denoted $N^{r}(S)$ (or simply $\left.N^{r}\right), 2 N^{r}$ is the number of $r$-cells.

On a cell complex there is a natural scalar product,

$$
\left(s, s^{\prime}\right)=1 \quad \text { if } \quad s=s^{\prime} \text { and } 0 \text { otherwise. }
$$

This gives for $r$-chains,

$$
\left(c, c^{\prime}\right)=\sum_{s_{r}} m_{s_{r}} m_{s_{r}}^{\prime} \quad\left(c^{\prime}=\sum_{s_{r}} m_{s_{r}}^{\prime} \cdot s_{r}\right) .
$$

One defines similarly the scalar product of a $G$ valued $r$-chain with an integral chain [if $m \in G, k \in Z, k m=m+m+\ldots+m$ ( $k$ times)]. Hence the scalar product $\left(c_{r}, s_{r}\right)$ of the chain $c_{r}$ and the integral chain $s_{r}$ is the value $c_{r}\left(s_{r}\right)$ that takes the chain $c_{r}$ on the $r$-cell $s_{r}$.

The boundary of an $r$-cell is an integral chain defined as

$$
\partial s_{r}=\sum_{s_{r-1}} I\left(s_{r}, s_{r-1}\right) s_{r-1} .
$$

The coboundary of an $r$-cell is the following integral $r+1$ chain,

$$
\partial^{*} s_{r}=\sum_{s_{r+1}} I\left(s_{r+1}, s_{r}\right) s_{r+1} .
$$

Notice that $I\left(s_{r}, s_{r-1}\right)=\left(\partial s_{r}, s_{r-1}\right)=\left(s_{r}, \partial^{*} s_{r-1}\right)$.

By linearity the boundary operator maps, $r$-chain into $r-1$ chain and the coboundary operator maps $r$-chain into $r+1$ chain and

$$
\left(\partial c_{r}, c_{r-1}\right)=\left(c_{r}, \partial^{*} c_{r-1}\right) .
$$

A cell complex $S^{\prime}$ is said to be a cell subcomplex of a cell complex $S$ if every element of $S^{\prime}$ is an element of $S$, every two elements $s_{r}, S_{r-1}$ of $S^{\prime}$ have the same incidence number in $S$ as they do in $S^{\prime}$, and every pair of opposites in $S^{\prime}$ is a pair of opposite elements in $S$. A cell subcomplex of $S^{\prime}$ is said to be closed (respectively open) if it contains with every cell also the cells of its boundary (respectively coboundary). We denote $\bar{S}^{\prime}$ the closure of $S^{\prime}$, i.e. the cell complex obtained by adding to each element its boundary cells.

The lattice $\mathbb{Z}^{2}$ may be considered as a cell complex denoted $S\left(\mathbb{Z}^{2}\right)$-0-cells are vertices, 1-cell links and 2-cells plaquettes. The orientation is the usual one and the incidence number takes the values \pm 1 if $s_{r-1}$ belongs to the boundary of $s_{r}$ with respect to the relative orientation and the value 0 otherwise. The boundary and coboundary operation are illustrated in the following picture: 


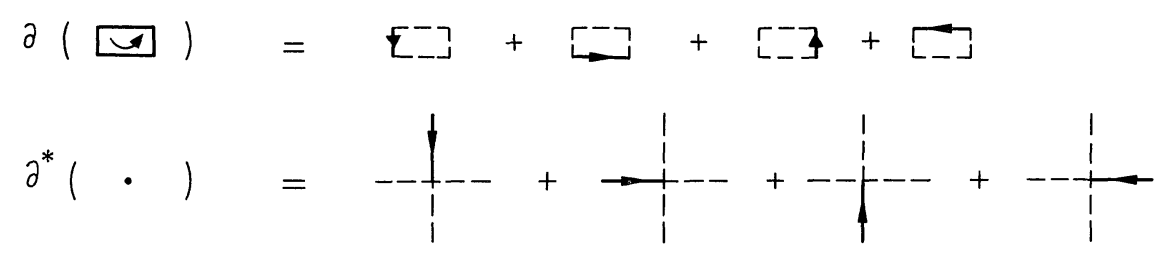

Consider now a cell subcomplex $S$ of $S\left(\mathbb{Z}^{2}\right)$, restrict the incidence function to this subset [then the boundary operation does not coincide with the same operation in $\left.S\left(\mathbb{Z}^{2}\right)\right]$. $S$ will be an $a$-complex if it verifies (2.1). In particular closed and open cell subcomplex are $a$-complex. $\Omega$ being a subset of $\mathbb{Z}^{2}$, we define closed subcomplexes of $S\left(\mathbb{Z}^{2}\right)$ as follows:

$$
S(\Omega), \Omega \subset \mathbb{Z}^{2}: S(\Omega)=\left\{S^{0}(\Omega), S^{1}(\Omega), S^{2}(\Omega)\right\},
$$

where

$$
\mid \begin{aligned}
& S^{0}(\Omega)=\left\{s_{0} \in S\left(\mathbb{Z}^{2}\right) / s_{0}= \pm x, x \in \mathbb{Z}^{2}\right\} \\
& S^{1}(\Omega)=\left\{s_{1} \in S\left(\mathbb{Z}^{2}\right) / \begin{array}{l}
\text { all the } s_{0} \text { such that }\left(\partial s_{1}, s_{0}\right) \neq 0 \\
\text { belongs to } S^{0}(\Omega)
\end{array}\right\} \\
& S^{2}(\Omega)=\left\{s_{2} \in S\left(\mathbb{Z}^{2}\right) / \begin{array}{l}
\text { all the } s_{1} \text { such that }\left(\partial s_{2}, s_{1}\right) \neq 0 \\
\text { belongs to } S^{1}(\Omega)
\end{array}\right\} .
\end{aligned}
$$

Let

$$
\mid \begin{aligned}
& S^{b}(\Omega)=\left\{s_{1} \in S(\Omega) / \exists s_{2} \in S\left(\mathbb{Z}^{2}\right) / S(\Omega) \text { s.t. }\left(\partial s_{2}, s_{1}\right) \neq 0\right\} \\
& S^{c}(\Omega)=\left\{s_{1} \in S\left(\mathbb{Z}^{2}\right) / S(\Omega) / \exists s_{0} \in S(\Omega) \text { s.t. }\left(\partial s_{1}, s_{0}\right) \neq 0\right\} .
\end{aligned}
$$

We define the boundary cell complex of $S(\Omega)$ denoted $\mathscr{B}_{S(\Omega)}$ as the closure of $S^{b}(\Omega)$, and the coboundary cell complex of $S(\Omega)$ denoted $\mathscr{C}_{S(\Omega)}$ as the cell complex obtained from $S^{c}(\Omega)$ by adding to each element of $S^{c}(\Omega)$ its coboundary cells.

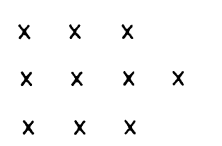

$\Omega$

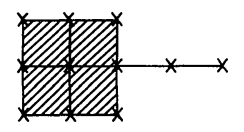

$\mathrm{S}(\Omega)$

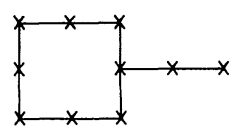

$B_{\mathrm{S}(\Omega)}$

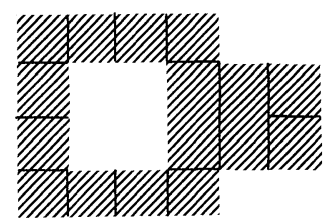

$\varphi_{S(\Omega)}$

In the following we shall consider only 2-dimensional complexes and shall denote respectively $v, l, p$ the 0 -cells, 1 -cells and 2 -cells.

\section{II.2. Definitions and Main Results}

We refer the reader to [10] for a general review on the Potts model. To introduce the 2-dimensional rectangular $q$-states Potts model one associates to a cell subcomplex $S$ of $S\left(\mathbb{Z}^{2}\right)$ and to any 0 -chain $c$ of $C^{0}\left(S, \mathbb{Z}_{q}\right)$ (i.e. a configuration), the hamiltonian

$$
H_{S}(c)=-\sum_{\ell \in S}\left(\delta_{(c, \partial \ell), 0}-1\right)
$$


The boundary operation is restricted to the complex-S - the sum is only over positively oriented links and $\delta$ is the Kronecker symbol $\left(\delta_{m, m^{\prime}}=1\right.$ if $m=m^{\prime}$ and 0 otherwise).

The partition function associated to $S$ is given by

$$
\Xi_{S}(\beta)=\sum_{c \in C^{0}\left(S, \mathbb{Z}_{q}\right)} e^{-\beta H_{S}(c)} .
$$

The boundary conditions (b.c.) in (2.9) are given by the geometry of the complex $S$ : if $S$ is open we have the usual " 0 " b.c., and if $S$ is closed we have the so-called free (" $f$ ") b.c. In the following we will be interested in more general boundary conditions, so for the closed complexes $S(\Omega)$ we define a characteristic function on the 0-chain of $\mathscr{B}_{S(\Omega)}\left(\right.$ for example $\left.\chi_{(c)}^{0}=\prod_{v \in \mathscr{B}_{S}} \delta_{(c, v), 0}, \chi_{(c)}^{f}=1\right)$, and the partition
functions

$$
\Xi_{S}^{\text {b.c. }} \cdot(\beta)=\sum_{c \in C^{0}\left(S, \mathbb{Z}_{q}\right)} e^{-\beta H_{S}(c)} \chi^{\text {b.c. }}(c) .
$$

For every measurable function $g$ with respect to the conditional Gibbs density $\left(\Xi_{S}^{\text {b.c. }}(\beta)\right)^{-1} \exp \left\{-\beta H_{S}(c)\right\} \chi_{(c)}^{\text {b.c. }}$, we introduce its expectation

$$
\langle g\rangle_{S}^{\text {b.c. }}(\beta)=\left(\Xi_{S}^{\text {b.c. }}(\beta)\right)^{-1}[g]_{S}^{\text {b.c. }}(\beta)=\left(\Xi_{S}^{\text {b.c. }}(\beta)\right)^{-1} \sum_{c \in C^{0}\left(S, \mathbb{Z}_{q}\right)} g e^{-\beta H_{S}(c)} \chi_{(c)}^{\text {b.c. }} .
$$

Let \langle\rangle$^{\text {b.c. }}(\beta)$ denotes the infinite volume limit of \langle\rangle$_{S(\Omega)}^{\text {b.c. }}(\beta)$ when $\Omega \uparrow \mathbb{Z}^{2}$ according to the D.L.R. equations [20].

These limits are uniquely defined for " 0 " and "/ " b.c. (this follows from correlation inequalities). Among the interesting expectations we consider

(i) the magnetisation (up to a constant)

$$
M(\beta)=\left\langle\delta_{(c, v), 0}-\frac{1}{q}\right\rangle^{0}(\beta),
$$

(ii) the long range order (two point function),

$$
\left\langle\delta_{\left(c, v-v^{\prime}\right), 0}-\frac{1}{q}\right\rangle^{f}(\beta)
$$

where $v$ and $v^{\prime}$ are positively oriented,

(iii) the mass gap or inverse correlation length,

$$
m(\beta)=\lim _{d\left(v, v^{\prime}\right) \rightarrow \infty}-\frac{1}{d\left(v, v^{\prime}\right)} \ln \left[\left\langle\delta_{\left(c, v-v^{\prime}\right), 0}-\frac{1}{q}\right\rangle^{f}(\beta)\right],
$$

where $d\left(v, v^{\prime}\right)$ is the euclidean distance between the vertex $v$ and $v^{\prime}$. It follows from correlation inequalities that this limit exists.

Theorem 2.1. The 2-dimensional $q$ states Potts model exhibits a first order phase transition at the self dual temperature $\beta_{t}(q)=\log (\sqrt{q}+1)$ if $q>25$ : the derivative of the free energy with respect to $\beta$ and the magnetisation are discontinuous at $\beta_{t}(q)$.

a) At $\beta_{t}(q)$ there are at least $q+1$ states \langle\rangle$^{\alpha}, \alpha \in\{0, \ldots, q-1\}$ and \langle\rangle$^{f}$ such that

$$
\begin{gathered}
\left.\begin{array}{c}
\left\langle\delta_{(c, \partial \ell), 0}-\lambda / 2\right\rangle^{\alpha}\left(\beta_{t}\right)>0 \\
\left\langle\delta_{(c, \partial \ell), 0}-\lambda / 2\right\rangle^{f}\left(\beta_{t}\right)<0
\end{array}\right\} \lambda=1+1 / \sqrt{q} . \\
M\left(\beta_{t}\right)>0
\end{gathered}
$$


b) For $\beta<\beta_{t}(q)$ the magnetisation $M(\beta)$ is zero.

It had been proven by Hintermann, Kunz, and $\mathrm{Wu}[15]$ that the free energy of the 2-dimensional $q$-states Potts model is analytic in $\beta$ except possibly at $\beta_{t}$ provided $q \geqq 4$. So the structure of translation invariant Gibbs states can then be known in this case [14]: for $\beta \neq \beta_{t}$ every translation invariant state is a linear combination of the states \langle\rangle$^{\alpha}$. It then follows from Theorem $2 \mathrm{~b}$ that for $\beta<\beta_{t}$ there is only one translation invariant state. ${ }^{1}$

Once one knows that several phases coexist at a given temperature, an interesting problem is the existence or not of a surface tension between these phases. One way to define the surface tension microscopically is to consider a partition function with "mixed" b.c. $[7,11]$. More precisely consider the following rectangular box:

$$
\Omega_{\mathrm{L} \cdot \mathrm{M}}=\left\{x=\left\{x^{1}, x^{2}\right\} \in \mathbb{Z}^{2} / 0 \leqq x^{1} \leqq L,-M-1 \leqq x^{2} \leqq M\right\} .
$$

The surface tension between two ordered phases is defined by [5]:

$$
\tau^{\alpha_{1}, \alpha_{2}}(\beta)=\lim _{\substack{M \rightarrow \infty \\ L \rightarrow \infty}}-\frac{1}{L} \ln \frac{\Xi_{S(\Omega)}^{\alpha_{1}, \alpha_{2}}(\beta)}{\left(\Xi_{S(\Omega)}^{\alpha_{1}}(\beta) \Xi_{S(\Omega)}^{\alpha_{2}}(\beta)\right)^{1 / 2}},
$$

where

$$
\chi^{\alpha_{1} \alpha_{2}}(c)=\prod_{v \in \mathscr{B}_{S}^{+}} \delta_{(c, v), \alpha_{1}} \prod_{v \in \mathscr{B}_{S(\Omega)} / \mathscr{B}_{S}^{+}} \delta_{(c, v), \alpha_{2}} .
$$

$\mathscr{B}_{S}^{+}$is the cell subcomplex of $\mathscr{B}_{S(\Omega)}$ such that its cells have non-negative coordinates. The product in $\chi^{\alpha_{1} \alpha_{2}}$ is over positively oriented 0 -cells.

The surface tension between an ordered and the disordered (free) phase is defined by:

$$
\tau^{0 f}(\beta)=\lim _{\substack{M \rightarrow \infty \\ L \rightarrow \infty}}-\frac{1}{L} \ln \frac{\Xi_{S(\Omega)}^{0 f}(\beta)}{\left(\Xi_{S(\Omega)}^{0}(\beta) \Xi_{S(\Omega)}^{f}(\beta)\right)^{1 / 2}},
$$

where

$$
\chi^{0 f}(c)=\prod_{v \in \mathscr{B}_{S}^{+}} \delta_{(c, v), 0}
$$

Theorem 2.2. For $q>\mu^{4}$ with $\mu=2,7$ (the self avoiding constant), we have the following properties:

a) $\tau^{\alpha_{1}, \alpha_{2}}(\beta)$ is strictly positive for $\beta \geqq \beta_{t}(q), \alpha_{1} \neq \alpha_{2} \in\{0, \ldots, q-1\}$ :

$$
\tau^{\alpha_{1} \alpha_{2}}(\beta) \geqq 2\left\{\frac{1}{4} \ln q-\ln \mu\right\} .
$$

b) $\tau^{0, f}(\beta)$ is strictly positive at $\beta_{t}(q)$ :

$$
\tau^{0 f}(\beta) \geqq \frac{1}{4} \ln q-\ln \mu .
$$

We believe that $\tau^{0 f}(\beta)$ is non-zero only at $\beta_{t}$.

1 We have been informed that Martirosian investigates this problem for general Potts models [30] 
The surface tension between two ordered phases can be compared to the mass gap using duality transformation and correlation inequalities.

$$
m(\beta) \leqq \frac{1}{q} \sum_{\alpha=1}^{q-1} \tau^{0 \alpha}\left(\beta^{*}\right) .
$$

The following theorem shows when $q$ is large the discontinuity of the mass gap at the transition point $\beta_{t}$ contrary to the Ising model $(q=2)$, where the mass gap is continuous,

Theorem 2.3. If $q>\mu^{4}$,

$$
\begin{gathered}
m(\beta)>2\left(\frac{1}{4} \ln q-\ln \mu\right) \quad \text { if } \quad \beta \leqq \beta_{t}(q), \\
m(\beta)=0 \quad \text { if } \quad \beta>\beta_{t}(q) .
\end{gathered}
$$

We describe in the following picture the expected phase diagram of the 2-dimensional Potts model which is presumably typical of models with large entropy.

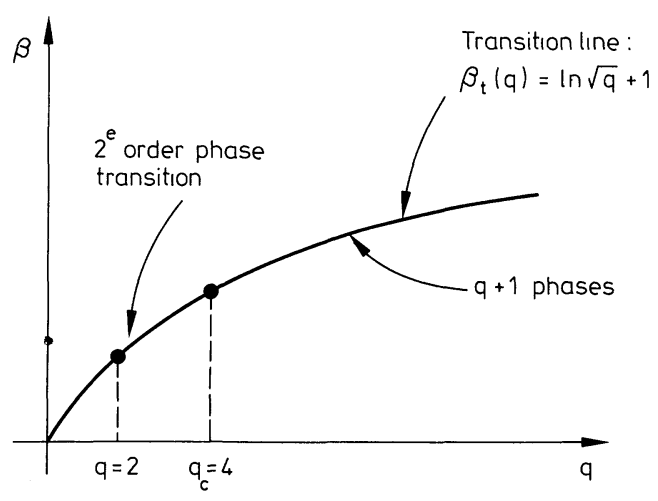

Remark 2.4. Our results are obtained for $q$ integer, but it is believed that they can be extended to $q$ real.

\section{Duality Transformation}

We refer the reader to $[26,27]$ for the duality in abelian lattice systems.

\section{III.1. Dual Lattice, Dual Complex}

Let $S$ be a 2-dimensional cell complex, the cell complex $S^{*}$ is said to be dual of $S$ if there is a one to one correspondence,

$$
S_{r} \leftrightarrow s_{2-r}^{*},
$$

between the $r$-cells $S_{r}$ of $S$ and the $2-r$-cells $s_{2-r}^{*}$ of $S^{*}$ such that the incidence numbers satisfy the relation

$$
I\left(s_{2-r+1}^{*}, s_{2-r}^{*}\right)=I\left(s_{r}, s_{r-1}\right) .
$$

The lattice

$$
\left(\mathbb{Z}^{2}\right)^{*}=\left\{\left(x^{1}+\frac{1}{2}, x^{2}+\frac{1}{2}\right) / x^{1} \in \mathbb{Z}, x^{2} \in \mathbb{Z}\right\}
$$


is the dual lattice of $\mathbb{Z}^{2}$. For any cell subcomplex $S$ of $S\left(\mathbb{Z}^{2}\right)$ there is a dual complex $S^{*}$ which is a subcomplex of $S\left(\left(\mathbb{Z}^{2}\right)^{*}\right)$.

The correspondence (3.1) is shown in the following picture:
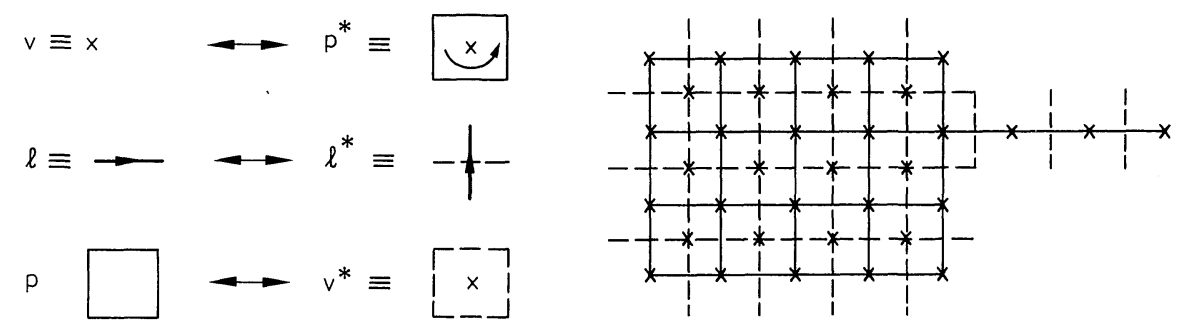

Notice that if $S$ is closed, $S^{*}$ is open and

$$
\mathscr{C}_{S}^{*}=\mathscr{B}_{\overline{S^{*}}} \quad \text { if } \quad S=S(\Omega) .
$$

We introduce the mapping $*$ (Hodge operation) which sends $C^{r}\left(S, \mathbb{Z}_{q}\right)$ onto $C^{2-r}\left(S^{*}, \mathbb{Z}_{q}\right)$

$$
\left(* c_{r}, s_{2-r}^{*}\right)=\left(c_{r}, s_{r}\right)
$$

It follows that

$$
* \partial * c_{r}=\partial * c_{r} * \partial c_{r}=\partial^{*} * c_{r}
$$

Therefore the mapping $*$ determines an isomorphism between

(i) The group of $\mathbb{Z}_{q}$-valued $r$-cycles of $S: Z_{r}\left(S, \mathbb{Z}_{q}\right)=\left\{c \in C^{r}\left(S, \mathbb{Z}_{q}\right) / \partial c=0\right\}$ and the group of $\mathbb{Z}_{q}$-valued $2-r$ cocycles of $S^{*}$ :

$$
Z^{2-r}\left(S^{*}, \mathbb{Z}_{q}\right)=\left\{c \in C^{2-r}\left(S^{*}, \mathbb{Z}_{q}\right) / \partial^{*} c=0\right\} .
$$

(ii) The group of $\mathbb{Z}_{q}$-valued $r$-boundary: $B_{r}\left(S, \mathbb{Z}_{q}\right)=\left\{c \in C^{r}\left(S, \mathbb{Z}_{q}\right) \mid c=\partial c_{r+1}\right\}$ and the group of $\mathbb{Z}_{q}$-valued $2-r$ coboundary of $S^{*}$

$$
B^{2-r}\left(S^{*}, \mathbb{Z}_{q}\right)=\left\{c \in C^{2-r}\left(S^{*}, \mathbb{Z}_{q}\right) / c=\partial^{*} c_{r-1}\right\} .
$$

Notice that since $\partial \partial=0$ [this follows from (3.1)] every boundary is a cycle: the converse is not true in general and the factor groups $H_{r}=Z_{r} / B_{r}, H^{r}=Z^{r} / B^{r}$ are called respectively $\mathbb{Z}_{q}$-valued homology and cohomology group. The rank of $H_{r}(S, \mathbb{Z})$ [denoted $H_{r}(S)$ ] is a topological invariant called $r$-betti number, denoted $\pi^{r}(S)$. We have $\pi^{r}(S)=\pi^{2-r}\left(S^{*}\right)$.

\section{III.2. Duality Transformation}

We assume that $S$ is an $a$-complex. To derive the duality relation for the partition function (2.9) we first consider the Fourier expansion of $e^{\beta \delta}$,

$$
\begin{aligned}
e^{\beta \delta_{(c, \partial \ell), 0}} & =1+\left(\mathrm{e}^{\beta}-1\right) \delta_{(c, \partial \ell), 0} \\
& =\sum_{m_{\ell}=0}^{q-1}\left(\delta_{m_{\ell}, 0}+\frac{e^{\beta}-1}{q}\right) \exp \left\{\frac{2 i \pi}{q} m_{\ell}(c, \partial \ell)\right\} \\
& =\frac{e^{\beta}-1}{q} \sum_{m_{\ell}=0}^{q-1}\left(1+\frac{q}{e^{\beta}-1}\right)^{\delta_{m_{\ell}, 0}} \exp \left\{\frac{2 i \pi}{q} m_{\ell}\left(\partial^{*} c, \ell\right)\right\} .
\end{aligned}
$$


One defines $\beta^{*}$ by

$$
\left(e^{\beta}-1\right)\left(e^{\beta^{*}}-1\right)=q
$$

and $c_{1}=\sum_{\ell \in S} m_{\ell} \cdot \ell$, then

$$
\Xi_{S}(\beta)=e^{-\beta N^{1}(S)}\left(\frac{e^{\beta}-1}{q}\right)^{N^{1}(S)} \sum_{\substack{c \in C^{0}\left(S, \mathbb{Z}_{q}\right) \\ c_{1} \in C^{1}\left(S, \mathbb{Z}_{q}\right)}} \exp \beta^{*}\left(\sum_{\ell} \delta_{\left(c_{1}, \ell\right), 0}\right) \exp \frac{2 i \pi}{q}\left(c_{1}, \partial c\right) .
$$

We use the relation (2.7) and sum over the elements of $C^{0}\left(S, \mathbb{Z}_{q}\right)$,

$$
\begin{aligned}
\Xi_{S}(\beta) & =e^{-\beta N^{1}}\left(\frac{e^{\beta}-1}{q}\right)^{N^{1}} q^{N^{0}} \sum_{c_{1} \in C^{\prime}\left(S, \mathbb{Z}_{q}\right)} \prod_{v \in S} \delta_{\left(\partial c_{1}, v\right), 0} \exp \beta^{*}\left(\sum_{\ell} \delta_{\left(c_{1}, \ell\right), 0}\right) \\
& =e^{-\beta N^{1}}\left(\frac{e^{\beta}-1}{q}\right)^{N^{1}} q^{N^{0}} \sum_{z \in Z_{1}\left(S, \mathbb{Z}_{q}\right)} \exp \beta^{*}\left(\sum_{\ell} \delta_{(z, \ell), 0}\right) .
\end{aligned}
$$

If $S$ is closed then it is torsion free (this follows from Alexander's theorem [17]). If one assumes that $\pi^{1}(S)=0$ (i.e. there is no "hole" in $S$ ), then $Z_{1}\left(S, \mathbb{Z}_{q}\right)=B_{1}\left(S, \mathbb{Z}_{q}\right)$. Since $\pi^{2}(S)=0$, we have

$$
\Xi_{S}(\beta)=e^{-\beta N^{1}}\left(\frac{e^{\beta}-1}{q}\right)^{N^{1}} q^{N^{0}} \sum_{c_{2} \in C^{2}\left(S, \mathbb{Z}_{q}\right)} \exp \beta^{*}\left(\sum_{\ell} \delta_{\left(\partial c_{2}, \ell\right), 0}\right)
$$

Taking into account the correspondence (3.1) and the isomorphism * between $C^{2}\left(S, \mathbb{Z}_{q}\right)$ and $C^{0}\left(S^{*}, \mathbb{Z}_{q}\right)$, we obtain

$$
\Xi_{S}(\beta)=e^{\left(\beta^{*}-\beta\right) N^{1}(S)}\left(\frac{e^{\beta}-1}{q}\right)^{N^{1}(S)} q^{N^{0}(S)} \Xi_{S^{*}}\left(\beta^{*}\right) .
$$

Notice that $\Xi_{S^{*}}^{0}\left(\beta^{*}\right)=\Xi_{S^{*}}(\beta)$.

In the following we will be interested in the self dual line defined by $\beta_{t}(q)$ $=\ln (\sqrt{q}+1)$. This line is obtained by letting $\beta=\beta^{*}$ in (3.6).

We now prove that the duality factor

$$
\Xi_{S}\left(\beta_{t}\right)\left(\Xi_{S^{*}}\left(\beta_{t}\right)\right)^{-1}=q^{N^{0}(S)-\frac{N^{1}(S)}{2}}
$$

is a boundary term.

Lemma 3.1. Let $\Omega \subset \mathbb{Z}^{2}$ and $S=S(\Omega)$, then:

$$
N^{0}(S(\Omega))-\frac{N^{1}(S(\Omega))}{2}=\frac{N^{1}\left(\mathscr{C}_{S(\Omega)}\right)}{4} .
$$

$\mathscr{C}_{S(\Omega)}$ is the coboundary complex of the complex $S(\Omega)$.

\section{Proof. Let}

$$
J(S, v)=\{\text { number of positively oriented } 1 \text {-cell } \ell \text { of } S \text { such that }(\partial \ell, v) \neq 0\} .
$$

Then since every 1 -cell contains two 0 -cells in its boundary

$$
N^{1}(S)=\sum_{v \in S} \frac{J(S, v)}{2},
$$


where the sum is restricted over positively oriented 0-cells. Then

$$
N^{0}(S)-\frac{N^{1}(S)}{2}=\sum_{v \in S}\left(1-\frac{J(s, v)}{4}\right)=\frac{1}{4} \sum_{\substack{v \in S: \\ J(S, v) \neq 4}}(4-J(S, v)) .
$$

For each $v$ in $S$ such that $J(S, v) \neq 4,4-J(S, v)$ is the number of positively oriented 1 -cells $\ell$ in $S\left(\mathbb{Z}^{2}\right) / S$ such that $(\partial \ell, v) \neq 0$. Then

$$
\sum_{v \in S}(4-J(S, v))=N^{1}\left(\mathscr{C}_{S}\right)
$$

This ends the proof of the lemma.

Proposition 3.1. Let $\Omega \subset \mathbb{Z}^{2}$ and assume that the complex $S=S(\Omega)$ has trivial homology. Let $L, L_{1}, L_{2}$ be 1-dimensional cell subcomplexes of $S$ such that $L_{1} \cap L_{2}=\emptyset$ :

$$
\begin{gathered}
\Xi_{S}^{f}\left(\beta_{t}\right)=q^{\frac{N^{1}\left(\mathscr{C}_{S}\right)}{4}} \Xi \overline{S^{*}}\left(\beta_{t}\right), \\
{\left[\prod_{\ell \in L_{1}}\left(1-\delta_{\ell}\right) \prod_{\ell \in L_{2}} \delta_{\ell}\right]_{S}^{f}\left(\beta_{t}\right)=q^{\frac{N^{1}\left(\mathscr{C}_{S}\right)}{4}}\left[\prod_{\ell * L_{1}^{*}}\left(\delta_{\ell^{*}}-\frac{1}{\sqrt{q}}\right) \prod_{\ell^{*} \in L_{2}^{*}}\left(\lambda-\delta_{\ell^{*}}\right)\right]_{\overline{S^{*}}}^{0}\left(\beta_{t}\right),} \\
\left\langle\prod_{\ell \in L}\left(\delta_{\ell}-\frac{\lambda}{2}\right)\right\rangle_{S}^{f}\left(\beta_{t}\right)=(-1)^{N^{1}(L)}\left\langle\prod_{\ell * L^{*}}\left(\delta_{\ell^{*}}-\frac{\lambda}{2}\right)\right\rangle_{\overline{S^{*}}}^{0}\left(\beta_{t}\right) .
\end{gathered}
$$

Hereafter we use the abbreviation $\delta_{\ell}$ for $\delta_{(c, \partial \ell), 0}$ when there is no possible confusion.

Proof. The first statement follows from (3.7) and Lemma 3.1. For the correlations we use $\delta_{\ell} e^{\beta\left(\delta_{\ell}-1\right)}=\delta_{\ell},\left(1-\delta_{\ell}\right) e^{\beta \delta_{\ell}}=1-\delta_{\ell}$ and proceed as for the partition function.

Proposition 3.1 shows clearly the analogy between the spin flip transformation in the Ising model and the duality transformation in the Potts model for which the free phase is sent into the ordered one with an energy cost proportional to the boundary. These relations are the analogous of the usual symmetry in the $\sigma$ variables for the Ising model. At non-zero $\beta^{*}-\beta$ we obtain a situation similar to an Ising model with magnetic field. In fact the Lee-Yang theorem had been proved in this case for $q \geqq 4[15]$.

\section{Contour Estimates}

We define the contours for the Potts model analogously to those defined in [1]. Let $\Omega \subset \mathbb{Z}^{2}$ be a rectangular box, $S=S(\Omega)$ the associated cell complex and $c$ a $\mathbb{Z}_{q}$-valued 0 -chain of $C^{0}\left(S, \mathbb{Z}_{q}\right)$ (i.e. a configuration). We say that a link $l$ of $S$ is ordered (for $c$ ) if $(c, \partial \ell)=0$ (we draw it -), and that it is disordered if $(c, \partial \ell) \neq 0$ (we draw it $m$ ).

Assume that for a given configuration there exists an "Island of pure phase" i.e. a connected subcomplex $S^{\prime}$ of $S$ such that all the links of $S^{\prime}$ are ordered and all the links of the coboundary $\mathscr{C}_{S^{\prime}}$ of $S^{\prime}$ are disordered. The subcomplex $\mathscr{C}_{S^{\prime}}$ is called contour of the configuration $c$. 
Definition. A (geometric) contour $\Gamma$ is an open complex which is the coboundary complex of a closed connected complex called $E$ such that $E=S\left(\Omega_{E}\right) \Omega_{E} \subset \mathbb{Z}^{2}$. We only consider contour $\Gamma$ constructed from a given configuration $c$ of $C^{0}\left(S, \mathbb{Z}_{q}\right)$.

We let $I=S / E \cup \Gamma$. Then $\Gamma$ is the coboundary of the closed subcomplex $I$. Notice that $I=S\left(\Omega_{I}\right)$ with $\Omega_{I} \subset \mathbb{Z}^{2}$.

Moreover $\Gamma^{*}$ divides $\Omega$ into $\Omega_{E}$ and $\Omega_{I}$.

Definition. A contour is called elementary if $\Gamma^{*}$ has one connected component $\left(\pi^{0}\left(\Gamma^{*}\right)=1\right)$. (In the usual terminology of contours $\Gamma^{*}$ corresponds to a self avoiding closed walk.)

We define the set of configurations associated to the contour as

$$
C_{\Gamma}=\left\{c \in C^{0}\left(S, \mathbb{Z}_{q}\right) \mid \begin{array}{l}
\text { for every vertex } v \text { of } \mathscr{B}_{E}(c, v)=0 \\
\text { for every link } \ell \text { of } \Gamma(c, \partial \ell) \neq 0
\end{array}\right\} .
$$

(In the terminology of [1] $\Gamma$ is a precontour and $C_{\Gamma}$ an union of contours.) We call outer contour a contour such that $\mathscr{B}_{E} \supset \mathscr{B}_{S}$.

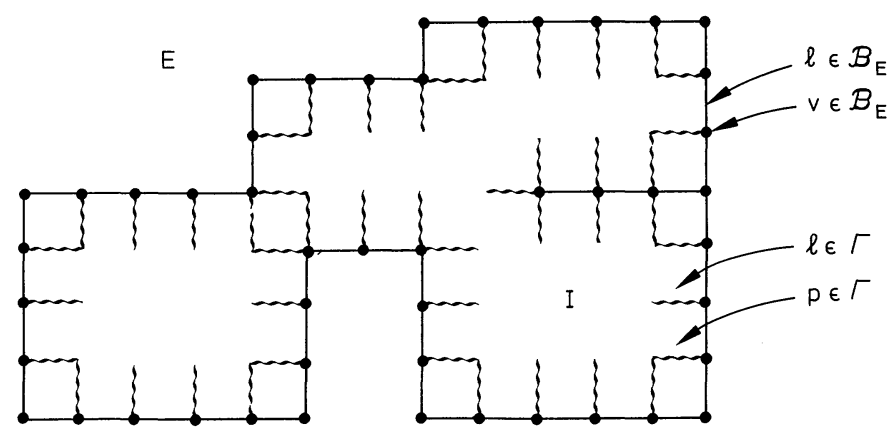

We define the probability of an outer contour by

$$
P_{\beta}(\Gamma)=\left(\Xi_{S}^{0}(\beta)\right)^{-1} \sum_{c \in C_{\Gamma}} e^{-\beta H_{S}(c)} \prod_{v \in \mathscr{B}_{S}} \delta_{(c, v), 0} .
$$

Proposition 4.1. Let $\Gamma$ be an (outer) contour. Then

$$
P_{\beta_{t}}(\Gamma) \leqq\left[\left(1+q^{-1 / 2}\right)^{4} q\right]^{-\frac{N^{1}(\Gamma)}{4}} .
$$

Remark. In [1] Kotecky and Shlossman have shown that the probability of contours are small in a large range of temperature but with periodic boundary conditions only. Our estimate can be obtained at $\beta_{t}$ but with arbitrary $y$ b.c. This can be compared with the Ising model with magnetic field $h$ when $\beta$ is large: the situation of [1] corresponds to the Ising model with free b.c. and our estimate corresponds to $h=0$.

Proof of the Proposition. Let

$$
\mathscr{B}_{I}^{\prime}=\left\{v \in \mathscr{B}_{I} / \exists \ell \in \Gamma \quad(\partial \ell, v)=0\right\} .
$$


Since for every $c$ in $C_{\Gamma}$ the links of $\Gamma$ are disordered, we have:

$$
\begin{aligned}
\Xi_{S}^{0}\left(\beta_{t}\right) P_{\beta_{t}}(\Gamma) & =e^{-\beta_{t} N^{1}(\Gamma)} \Xi_{E}^{0}\left(\beta_{t}\right) \sum_{c \in C^{0}\left(S, \mathbb{Z}_{q}\right)} e^{-\beta_{t} H_{I}(c)} \prod_{v \in \mathscr{B}_{I}^{\prime}}\left(1-\delta_{(c, v), 0}\right) \\
& \leqq e^{-\beta_{t} N^{1}(\Gamma)} \Xi_{E}^{0}\left(\beta_{t}\right) \Xi_{I}^{\not}\left(\beta_{t}\right),
\end{aligned}
$$

we compute the dual of the partition function $\Xi_{I}$.

By construction $I=S\left(\Omega_{I}\right)$ has trivial homology and we are in the hypothesis of Proposition 3.1. Therefore

$$
\Xi_{S}^{0}\left(\beta_{t}\right) P_{\beta_{t}}(\Gamma) \leqq e^{-\beta_{t} N^{1}(\Gamma)} q^{\frac{N^{1}\left(\mathscr{C}_{I}\right)}{4}} \Xi_{E}^{0}\left(\beta_{t}\right) \Xi_{I^{*}}^{0}\left(\beta_{t}\right) .
$$

Let $\left(\overline{I^{*}}\right)_{T}$ be the dual complex $\overline{I^{*}}$ translated by $1 / 2$ in the two directions. Then

$$
\begin{aligned}
\Xi_{S}^{0}\left(\beta_{t}\right) & \geqq \sum_{c \in C^{0}\left(S, \mathbb{Z}_{q}\right)} e^{-\beta_{t} H_{S}(C)} \prod_{\substack{v \in \mathscr{B}_{E} \\
v \in \mathscr{B}_{(\overline{I *}]_{T}}}} \delta_{(c, v), 0} \\
& =\Xi_{E}^{0}\left(\beta_{t}\right) \Xi_{\bar{I}^{*}}^{0}\left(\beta_{t}\right) .
\end{aligned}
$$

The proposition follows from the two last formulae by noticing that $\mathscr{C}_{I}=\Gamma$.

Remark. The computation works as well in the particular cases:

(i) $I$ is reduced to one point.

(ii) $\Xi_{I}$ is a one dimensional partition function.

(iii) I contains more than one connected component.

\section{Proof of Theorems}

\section{V.1. Proof of the Statement a of Theorem 2.1}

Let $\ell$ be some link in $S=S(\Omega)$, where $\Omega$ is some rectangular box. Then

$$
\begin{aligned}
\left\langle\delta_{(c, \partial \ell), 0}\right\rangle_{S}^{0}(\beta) & =\operatorname{Prob}\left\{(c, \partial \ell)=0 /(c, v)=0 \quad \text { if } \quad v \in \mathscr{B}_{S}\right\} \\
& =1-\operatorname{Prob}\left\{(c, \partial \ell) \neq 0 /(c, v)=0 \quad \text { if } \quad v \in \mathscr{B}_{S}\right\} .
\end{aligned}
$$

If we consider a configuration $c$ of $C^{0}(S, \mathbb{Z} q)$ such that $(c, v)=0$ if $v \in \mathscr{B}_{S}$ and $\ell$ is disordered, then necessarily there exists an elementary contour $\Gamma$ such that $c \in C_{\Gamma}$; $\Gamma^{*}$ enclose or contain $\ell$ and $N^{1}(\Gamma) \geqq 4$. Hence

$$
\begin{aligned}
& \operatorname{Prob}\{(c, \partial \ell) \neq 0 /(c, v)\}=0 \quad \text { if } \quad v \in \mathscr{B}_{S}, \beta=\beta_{t} \\
& \quad \leqq\left(\Xi_{S}^{0}\left(\beta_{t}\right)\right)^{-1} \sum_{\Gamma} \sum_{c \in C_{\Gamma}} e^{-\beta H_{S}(c)} \prod_{v \in \mathscr{B}_{S}} \delta_{(c, v), 0} \\
& \quad \leqq \sum_{\Gamma} P_{\beta_{t}}(\Gamma) .
\end{aligned}
$$

The sum is restricted over the elementary contour $\Gamma^{*}$ which contains or enclose the link $\ell$. We use the three way argument (the number of contours of length $k$ containing or enclosing a given link is bounded by $k \cdot \mu^{k-2}$ (where $\mu=2,7$ see [29])), the above relations and Proposition 4.1 to prove that for $q>25,\left\langle\delta_{\ell}\right\rangle^{0}\left(\beta_{t}\right)>\lambda / 2$. The formula for the magnetization is obtained in the same way: if we consider a configuration such that $(c, v)=0$ if $v \in \mathscr{B}_{S}$ and $\left(c, v_{0}\right) \neq 0$, then necessarily there exists an elementary contour $\Gamma$ such that $c \in C_{\Gamma} ; \Gamma^{*}$ enclose $v_{0}$. The second formula of Theorem 2.1 follows from the duality relation of Proposition 3.1. 


\section{V.2. Proof of the Statement a of Theorem 2.2}

Let $\Omega=\Omega_{L \cdot M}$ be the box defined in (2.15) and $S=S(\Omega)$. Consider the partition function $\Xi_{S}^{\alpha_{1}, \alpha_{2}}(\beta)$. For any configuration $c$ of $C^{0}(S, \mathbb{Z} q)$ such that $\chi^{\alpha_{1}, \alpha_{2}}(c)=1$, there exist two connected cell subcomplex $S_{1}, S_{2}$ such that $S_{1}=S\left(\Omega_{1}\right), S_{2}=S\left(\Omega_{2}\right)$ and

(i) The boundary $\mathscr{B}_{S_{1}}$ of $S_{1}$ contain $\mathscr{B}_{S}^{+}$, and for all positively oriented vertex $v$ in $\mathscr{B}_{S_{1}}(c, v)=\alpha_{1}$.

(ii) The boundary $\mathscr{B}_{S_{2}}$ of $S_{2}$ contains $\mathscr{B}_{S}^{-}\left(\mathscr{B}_{S}^{-}\right.$is the symmetric of $\mathscr{B}_{S}^{+}$with respect to the plane $x^{2}=-\frac{1}{2}$ ) and for all, positively oriented vertex $v$ in $\mathscr{B}_{S_{2}}(c, v)=\alpha_{2}$.

(iii) The links of $\mathscr{C}_{S_{1}}$ and the links of $\mathscr{C}_{S_{2}}$ are disordered.

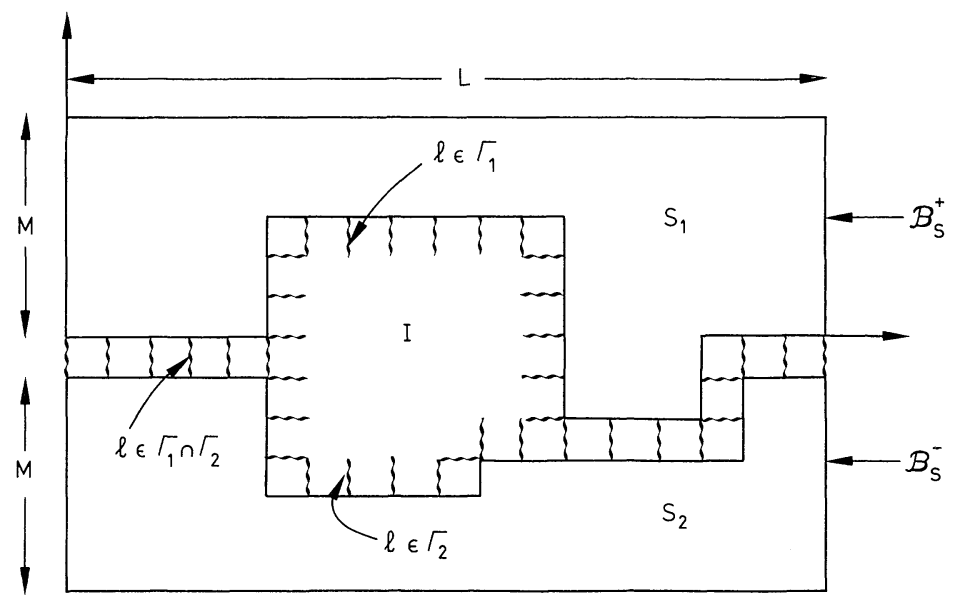

Let

$$
I=S / S_{1} \cup S_{2} \cup \mathscr{C}_{S_{1}} \cup \mathscr{C}_{S_{2}}, \quad \Gamma_{1}=\mathscr{C}_{S_{1}} \cap S, \quad \Gamma_{2}=\mathscr{C}_{S_{2}} \cap S
$$

for $i=1,2$,

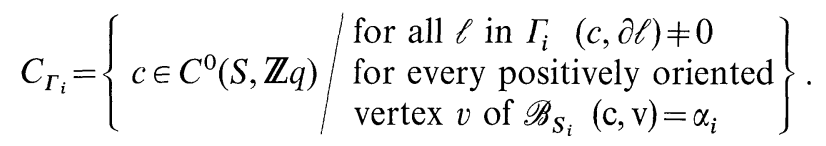

We define the weight of $C_{\Gamma_{1}} \cap C_{\Gamma_{2}}$ by:

Then

$$
\omega_{\beta}\left(C_{\Gamma_{1}} \cap C_{\Gamma_{2}}\right)=\left(\Xi_{S}^{\alpha_{1}}(\beta) \Xi_{S}^{\alpha_{2}}(\beta)\right)^{-1 / 2} \sum_{c \in C_{\Gamma_{1}} \cap C_{\Gamma_{2}}} e^{-\beta H_{S}(c)} .
$$

$$
\left(\Xi_{S}^{\alpha_{1}}(\beta) \Xi_{S}^{\alpha_{2}}(\beta)\right)^{-1 / 2} \Xi_{S}^{\alpha_{1}, \alpha_{2}}(\beta)=\sum_{\Gamma_{1}, \Gamma_{2}} \omega_{\beta}\left(C_{\Gamma_{1}} \cap C_{\Gamma_{2}}\right) .
$$

We now give an estimate on $\omega_{\beta_{t}}\left(C_{\Gamma_{1}} \cap C_{\Gamma_{2}}\right)$. For $c \in C_{\Gamma_{1}} \cap C_{\Gamma_{2}}$ all the links of $\Gamma_{1}$ and $\Gamma_{2}$ are disordered,

$$
\sum_{c \in C_{\Gamma_{1}} \cap C_{\Gamma_{2}}} e^{-\beta_{t} H_{S}(c)} \leqq e^{\left.-\beta_{t}\left(N^{1} / \Gamma_{1} \cap \Gamma_{2}\right)+N^{1}\left(\mathscr{G}_{I}\right)\right)} \Xi_{S_{1}}^{\alpha_{1}}\left(\beta_{t}\right) \Xi_{S_{2}}^{\alpha_{2}}\left(\beta_{t}\right) \Xi_{I}^{t}\left(\beta_{t}\right) .
$$


We compute the dual of the partition function $\Xi_{I}^{f}\left(\beta_{t}\right)$ and use

to obtain

$$
\left\{\begin{array}{c}
\Xi_{S_{1}}^{\alpha_{1}} \Xi_{S_{2}}^{\alpha_{2}} \Xi_{I^{*}}^{0}=\Xi_{S_{1}}^{0} \Xi_{S_{2}}^{0} \Xi_{I^{*}}^{0} \leqq \Xi_{S}, \\
N^{1}\left(\mathscr{C}_{I}\right)+2 N^{1}\left(\Gamma_{1} \cap \Gamma_{2}\right)=N^{1}\left(\Gamma_{1}\right)+N^{1}\left(\Gamma_{2}\right),
\end{array}\right.
$$

$$
\omega_{\beta_{t}}\left(C_{\Gamma_{1}} \cap C_{\Gamma_{2}}\right) \leqq e^{-\frac{\beta_{t}}{2}\left(N^{1}\left(\Gamma_{1}\right)+N^{1}\left(\Gamma_{2}\right)\right)} \leqq q^{-\frac{1}{4}\left(N^{1}\left(\Gamma_{1}\right)+N^{1}\left(\Gamma_{2}\right)\right)} .
$$

The contour $\Gamma_{i}, i=1,2$ have a "minimal length" $\left(N^{1}\left(\Gamma_{i}\right)\right)$ equal to $L$. It then follows from (5.3) and the three way argument that the left-hand side of (5.2) decreases exponentially with $L$ when $q>3^{4}$ and $\beta=\beta_{t}$. This proves the theorem for $\beta=\beta_{t}$. It follows from Ginibre's inequality $[18,19]$ that the surface tension is increasing in $\beta$. This extends the proof to $\beta>\beta_{t}$.

Remark. We think that for $\beta>\beta_{t}$ the typical configuration are such that the region $I$ is very thin and that for $\beta=\beta_{t}$ it becomes a drop.

\section{V.3. Proof of the Statement a of Theorem 2.3}

We first compute the dual of the mass-gap using the Fourier expansion of $\delta$,

$$
\begin{aligned}
m(\beta)= & \lim _{d\left(v, v^{\prime}\right) \rightarrow \infty}-\frac{1}{d\left(v, v^{\prime}\right)} \ln \sum_{\alpha=1}^{q-1}\left\langle e^{\frac{2 i \pi}{q} \alpha\left(c, v-v^{\prime}\right)}\right\rangle^{f}(\beta), \\
\left\langle e^{\frac{2 i \pi}{q} \alpha\left(c, v-v^{\prime}\right)}\right\rangle_{S}^{f}(\beta)= & \left(\Xi_{S^{*}}^{0}\left(\beta^{*}\right)\right)^{-1} \sum_{c \in C^{0}\left(S^{*} \mathbb{Z} q\right)} \\
& \cdot \exp \beta^{*} \sum_{\ell^{*} \in S^{*}}\left(\delta_{\left.\left(c, \partial \ell^{*}\right), \alpha^{*} h, \ell^{*}\right)}-1\right) \prod_{v^{*} \in \mathscr{B} \bar{S}^{*}} \delta_{\left(c, v^{*}\right), 0} .
\end{aligned}
$$

$h$ is an integral chain such that $\partial h=v-v^{\prime}$. For example assume that $v$ and $v^{\prime}$ are on the axis $x^{2}=0$. Let $\gamma$ be the set of links of $S$ on the axis $x^{2}=0$ and $\gamma^{\prime}$ be the subset of $\gamma$ between $v$ and $v^{\prime}$. We take $h=\sum_{\ell} m_{\ell} \cdot \ell$ with $m_{\ell}=1$ if $\ell \in \gamma^{\prime}$ and $\ell$ positively oriented and $m_{\ell}=0$ otherwise.

The proof is now a simple adaptation of that of Theorem 2.2, as it is clear in the preceding formulae; we have in this case open contours between the vertices $v$ and $v^{\prime}$; so we derive the following estimate:

$$
\left\langle e^{\frac{2 i \pi}{q} \alpha\left(c, v-v^{\prime}\right)}\right)_{s}^{f}\left(\beta_{t}\right) \leqq \operatorname{const}\left(\mu q^{-1 / 4}\right)^{2 d\left(v, v^{\prime}\right)} .
$$

This proves the theorem for $\beta=\beta_{t}$. It follows from Ginibre's inequality that the mass-gap is decreasing in $\beta$. This extends the proof to $\beta<\beta_{t}$.

\section{V.4. Proof of the Statement b of Theorem 2.3}

We have to prove

$$
\lim _{d\left(v, v^{\prime}\right) \rightarrow \infty}\left\langle\delta_{\left(c, v-v^{\prime}\right)}-\frac{1}{q}\right\rangle^{f}(\beta)>0 \quad \text { if } \quad \beta>\beta_{t} .
$$

It is known [20] that

$$
\lim _{\varepsilon \rightarrow 0}\left\langle\delta_{\left(c, v-v^{\prime}\right), 0}\right\rangle^{f}(\beta+\varepsilon) \geqq\left\langle\delta_{\left(c, v-v^{\prime}\right), 0}\right\rangle^{0}(\beta) .
$$


The state \langle\rangle$^{0}(\beta)$ is clustering, hence:

$$
\begin{gathered}
q\left\langle\delta_{\left(c, v-v^{\prime}\right), 0}-\frac{1}{q}\right\rangle^{0}(\beta)=\left\langle\sum_{r=1}^{q} \cos \left(\frac{2 \pi r}{q}\left(c, v-v^{\prime}\right)\right)\right\rangle^{0}(\beta) \\
\rightarrow \sum_{r=1}^{q}\left\langle\cos \frac{2 \pi r}{q}(c, v)\right\rangle^{0}(\beta)\left\langle\cos \frac{2 \pi r}{q}\left(c, v^{\prime}\right)\right\rangle^{0}(\beta) .
\end{gathered}
$$

Ginibre's inequality and Theorem 2.1a imply

$$
\begin{gathered}
\left\langle\cos \frac{2 \pi r}{q}(c, v)\right\rangle^{0}(\beta) \geqq 0, \\
M(\beta)=\frac{1}{q} \sum_{r=1}^{q}\left\langle\cos \frac{2 \pi r}{q}(c, v)\right\rangle^{0}(\beta)>0 \quad \text { if } \quad \beta \geqq \beta_{t} .
\end{gathered}
$$

The four last formulae implies (5.4) and hence the statement $\mathrm{b}$ of Theorem 3.

\section{V.5. Proof of the Statement b of Theorem 2.1}

We have from (5.5) and (5.6),

$$
\begin{aligned}
& \sum_{r=1}^{q}\left\langle\cos \frac{2 \pi r}{q}(c, v)\right\rangle^{0}(\beta)\left\langle\cos \frac{2 \pi r}{q}\left(c, v^{\prime}\right)\right\rangle^{0}(\beta) \\
& \leqq \lim _{d\left(v, v^{\prime}\right) \rightarrow \infty}\left\langle\delta_{\left(c, v-v^{\prime}\right)}-\frac{1}{q}\right\rangle^{f}(\beta+\varepsilon)
\end{aligned}
$$

for $\varepsilon$ positive arbitrary small. Since the mass gap $m(\beta)$ is non-zero for $\beta \leqq \beta_{t}$ (Theorem 2a) the limit in (5.9) is zero if $\beta+\varepsilon \leqq \beta_{t}$. Therefore the left-hand side of (5.9) is zero for $\beta<\beta_{t}$. We then use (5.7) to obtain

$$
\left\langle\cos \frac{2 \pi r}{q}(c, v)\right\rangle^{0}(\beta)=0 \quad \text { if } \quad \beta<\beta_{t} .
$$

Hence the magnetisation $M(\beta)$ is zero for $\beta<\beta_{t}$.

V.6. Proof of the Statement $\mathrm{b}$ of Theorem 2.3

Consider the partition function $\Xi_{S}^{0, f}(\beta)$, where $\Omega$ is the rectangular box $\Omega_{L \cdot M}$ defined in (2.15) and $S=S(\Omega)$.

For each configuration $c$ of $C^{0}(S, \mathbb{Z} q)$ such that $\prod_{v \in \mathscr{B}_{s}^{+}} \delta_{(c, v)}=1$, there exists a connected subcomplex $U$ such that, $U=S\left(\Omega_{u}\right): \Omega_{u} \subset \Omega ; \mathscr{B}_{U}$ contains $\mathscr{B}_{S}^{+}$, for all $v$ of $\mathscr{B}_{U}(c, v)=0$ and all the links of $\mathscr{C}_{U} \cap S$ are disordered. Let $D$ be the following subcomplex:

$$
D=S / U \cup\left(\mathscr{C}_{U} \cap S\right) \text { and } \Gamma=\mathscr{C}_{U} / \mathscr{C}_{S}^{+}
$$

where $\mathscr{C}_{S}^{+}$is the subset of $\mathscr{C}_{S}$ in the half plane $x^{2}>0$. Notice that there exists $c$ such that $D=\phi$ (see the picture below). 

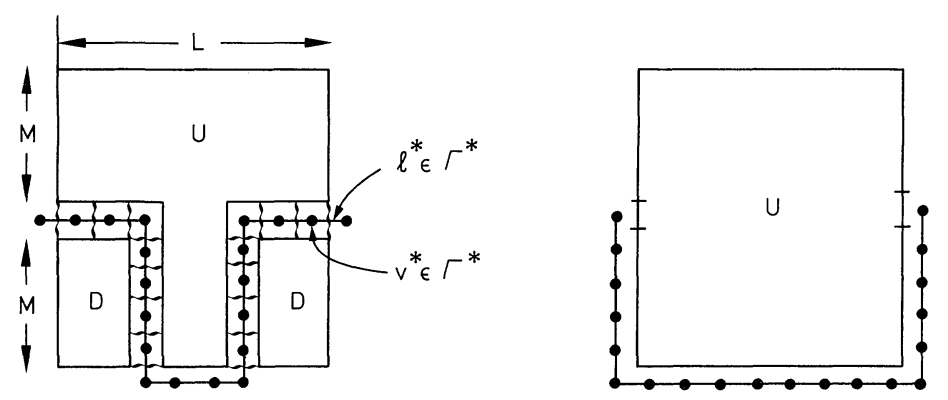

We define

$$
\begin{gathered}
C_{\Gamma}=\left\{c \in C^{0}(S, \mathbb{Z} q) \mid \begin{array}{l}
\text { for every vertex } v \text { of } \mathscr{B}_{U}(c, v)=0 \\
\text { for every link } \ell \text { of } \mathscr{C}_{U} \cap S \quad(c, \partial \ell) \neq 0
\end{array}\right\}, \\
\omega_{\beta}\left(C_{\Gamma}\right)=\left(\Xi_{S}^{0}(\beta) \Xi_{S}^{f}(\beta)\right)^{-1 / 2} \sum_{c \in C_{\Gamma}} e^{-\beta H_{S}(c)} .
\end{gathered}
$$

Then

$$
\frac{\Xi_{S}^{0 f}(\beta)}{\left(\Xi_{S}^{0}(\beta) \Xi_{S}^{f}(\beta)\right)^{1 / 2}}=\sum_{\Gamma} \omega_{\beta}\left(C_{\Gamma}\right)
$$

Proposition 5.1. With the above definitions $\omega_{\beta_{t}}\left(C_{\Gamma}\right) \leqq q^{-\frac{N^{1}(\Gamma)}{4}}$.

Proof. For any $c$ in $C_{\Gamma}$ the links of $\mathscr{C}_{U} \cap S$ are disordered.

Therefore,

$$
\omega_{\beta}\left(C_{\Gamma}\right) \leqq e^{-\beta N^{1}\left(\mathscr{C}_{U} \cap S\right)} \frac{\Xi_{U}^{0}(\beta) \Xi_{D}^{f}(\beta)}{\left(\Xi_{S}^{0}(\beta) \Xi_{S}^{f}(\beta)\right)^{1 / 2}}
$$

We compute the dual of the partition functions with free b.c. in the above formula. From Proposition 3.1 it follows.

$$
\omega_{\beta_{t}}\left(C_{\Gamma}\right) \leqq e^{-\beta_{t} N^{1}\left(\mathscr{C}_{U} \cap S\right)} q^{\frac{1}{4} N^{1}\left(\mathscr{C}_{D}\right)-\frac{1}{8}\left(\mathscr{C}_{S}\right)} \frac{\Xi_{U}^{0}\left(\beta_{t}\right) \Xi_{\bar{D}^{*}}^{0}\left(\beta_{t}\right)}{\left(\Xi_{S}^{0}\left(\beta_{t}\right) \Xi_{S^{*}}^{0}\left(\beta_{t}\right)\right)^{1 / 2}} .
$$

We have

$$
\begin{gathered}
\mathscr{C}_{U} \cap S=\mathscr{C}_{D} \cap S \\
\frac{N^{1}\left(\mathscr{C}_{S}\right)}{2}=\left[N^{1}(\Gamma)-N^{1}\left(\mathscr{C}_{D} \cap S\right)\right]+\left[N^{1}\left(\mathscr{C}_{D}\right)-N^{1}\left(\mathscr{C}_{D} \cap S\right)\right] .
\end{gathered}
$$

We will now prove that

$$
\Xi_{U}^{0}(\beta) \Xi_{\bar{D}^{*}}^{0}(\beta) \leqq\left(\Xi_{S}^{0}(\beta) \Xi_{S^{*}}^{0}(\beta)\right)^{1 / 2}
$$

For this we will use reflection positivity [21] with respect to a suitable plane 
$\left(x^{2}=-\frac{1}{2}\right)$. Let

$$
\begin{gathered}
\Omega^{\prime}=\left\{x=\left\{x^{1}, x^{2}\right\} \in \mathbb{Z}^{2} / 0 \leqq x^{1} \leqq L+1,-M-1 \leqq x^{2} \leqq M\right\}, \\
S^{\prime}=S\left(\Omega^{\prime}\right), \\
f=\prod_{v \in \mathscr{B}_{s}^{+}} \delta_{(c, v), 0} \prod_{v \in \mathscr{B}_{S^{\prime}}^{+}} \delta_{(c, v), 0}, \\
g=\prod_{v \in \mathscr{B}_{s^{\prime}}^{+}} \delta_{(c, v), 0}
\end{gathered}
$$

$\pi$ the plane $x^{2}=-\frac{1}{2}$.

We define $\theta_{\pi} F(v)=F \theta_{\pi}(v)$ (see [21]), where $\theta_{\pi}(v)$ is the symmetric of $v$ with respect to $\pi$. Since $\Omega^{\prime}$ is symmetric with respect to $\pi$ the hamiltonian $H_{S^{\prime}}$ defined in (2.8) is reflection positive with respect to $\pi$ (see [21] or [1]):

$$
\left[f \theta_{\pi} g\right]_{S^{\prime}}^{f}(\beta) \leqq\left(\left[f \theta_{\pi} f\right]_{S^{\prime}}(\beta)\left[g \theta_{\pi} g\right]_{S^{\prime}}(\beta)\right)^{1 / 2} .
$$

We have

$$
\left\{\begin{array}{c}
{\left[f \theta_{\pi} f\right]_{S^{\prime}}^{f}(\beta)=\Xi_{S}^{0}(\beta),} \\
{\left[g \theta_{\pi} g\right]_{S^{\prime}}^{f}(\beta)=\Xi_{S^{\prime}}^{0}(\beta) \leqq \Xi_{\bar{S}^{*}}^{0}(\beta) .}
\end{array}\right.
$$

Now let $\left(\overline{D^{*}}\right)_{T}$ the complex $\overline{D^{*}}$ translated by $+\frac{1}{2}$ in the two directions, then

$$
\left[f \theta_{\pi} g\right]_{S^{\prime}}^{f}(\beta) \geqq\left[\left(f \theta_{\pi} g\right) \prod_{\substack{v \in \mathscr{B}_{U} \\ v \in \mathscr{B}_{\left(\overline{D^{*}}\right) T}}} \delta_{(c, v), 0}\right]_{S^{\prime}}^{f}(\beta)=\Xi_{U}^{0}(\beta) \Xi_{\bar{D}^{*}}^{0}(\beta) .
$$

The above relations imply (5.14) and the proposition follows from (5.12), (5.13), (5.14).

Since the contour $\Gamma$ have a minimal length $L$ the statement b of Theorem 2.2 follows from Proposition 5.1 and (5.11). This ends the proofs of the theorems.

Remark. We can use this method to prove that another surface tension $\tau^{0, \mathrm{dis}}(\beta)$ is non-zero at $\beta_{t} ; \tau^{0 \text {,dis }}$ is defined similarly to $\tau^{0, f}[\operatorname{see}(2.17)]$ but with the boundary conditions respectively given by

$$
\chi_{(c)}^{\mathrm{dis}}=\prod_{\ell \in \mathscr{B}_{S}}\left(1-\delta_{(c, \partial \ell), 0}\right), \quad \chi_{(c)}^{0, \mathrm{dis}}=\prod_{v \in \mathscr{B}_{S}^{+}} \delta_{(c, v), 0} \prod_{\ell \in \mathscr{B} / \mathscr{B}+}\left(1-\delta_{(c, \partial \ell), 0}\right) .
$$

\section{Conclusion}

We want to emphasize that the results we have obtained for the two dimensional Potts model using the duality transformation are a particular case of a general theory describing these order disordered phase transitions just as the Peierls argument with the spin flip of the Ising model can be extended to a very general concept describing the phase coexistence at low temperature: the Pirogov Sinai theory. We want to give some possible generalizations.

1. The class of self dual model. (By this we mean that the hamiltonian after the duality transformation is identical up to the coupling constant to the original one.) A particularly interesting case is the 4 dimensional gauge model, where a similar argument works to prove the phase transition between ordered and disordered phase at $\beta_{t}$ and a discontinuity of the string tension at $\beta_{t}$ [23]. 
2. The models which are not self dual, a typical case of which is the 3dimensional Potts model. We strongly believe that our methods work also.

3. The duality transformation can be done on subgroups of $q$ with some appropriate hamiltonian we could obtain when $\beta$ increases the occurrence of a sequence of order disorder phase transitions.

\section{Appendix}

\section{A.1. Generalized Ginibre Inequality [18, 19]}

Let $\Omega=(1, \ldots, n)$ be a finite set of sites and associate to each site $i$ a spin $\sigma_{i}=\left(\cos \theta_{i}, \sin \theta_{i}\right), \theta_{i} \in[0,2 \pi]$. Let $\mathscr{M}_{\Omega}$ be the set of all $M=\left\{M_{1} \ldots M_{n}\right\}, M_{i}$ taking integer values, and $M \theta=\sum M_{i} \theta_{i}$. Let $J$ and $J^{\prime}$ be two real valued functions defined on $\mathscr{M}_{\Omega}$ and for each $M$ let $\psi_{M} \in[0,2 \pi]$. Consider the two measures

$$
\begin{gathered}
\mu(d \theta)=\Xi^{-1} \exp \left\{\sum_{M \in \mathcal{M}_{\Omega}} J(M) \cos M \theta\right\} d \theta, \\
\mu^{\prime}(d \theta)=\left(\Xi^{\prime}\right)^{-1} \exp \left\{\sum_{M \in \mathcal{M}_{\Omega}} J^{\prime}(M) \cos \left(M \theta+\psi_{M}\right)\right\} d \theta .
\end{gathered}
$$

We note the corresponding expectations by \langle\rangle\langle\rangle$^{\prime}$. We have, if $J(M) \geqq\left|J^{\prime}(M)\right|$, the following inequalities:

$$
\left.\begin{array}{l}
\langle\cos M \theta\rangle-\langle\cos M \theta-\varphi\rangle^{\prime} \geqq 0 \\
\langle\cos M \theta\rangle \geqq 0
\end{array}\right\} \text { for all } M \text {; for all } \varphi \in[0,2 \pi] .
$$

The above inequality applies to the Potts model by restricting the values $\theta_{i}$ to $\theta_{i}=2 k_{i} \pi / q$, this by adding an external interaction $\sum_{i} K \cos q \theta_{i}$ to $H_{\Omega}$ with $K \rightarrow \infty$.

Acknowledgements. We are very indebted to J. Bricmont, J. L. Lebowitz, S. Miracle-Sole, and C.-E. Pfister for helpful discussions and comments.

L. Laanait thanks the Université de Provence and the C.P.T. for financial support and kind hospitality.

\section{References}

1. Kotecky, R., Shlossman, S.B.: First order phase transitions in large entropy lattice models. Commun. Math. Phys. 83, 493-515 (1982)

2. Simon, B.: Correlation inequalities and the decay of correlation in ferromagnets. Commun. Math. Phys. 77, 111-126 (1980)

3. Sinai, Ya.G.: Theory of phase transitions: Rigorous results. London: Pergamon Press 1982

4. Fröhlich, J., Simon, B., Spencer, T.: Infrared bounds, phase transitions and continuous symmetry breaking. Commun. Math. Phys. 50, 79-85 (1976)

5. Bricmont, J., Lebowitz, J.L., Messager, A.: First order phase transitions in Potts and Ising systems. Phys. Lett. A 95, 169-172 (1983)

6. Griffiths, R.B.: Rigorous results and theorems in Domb-Green: phase transitions and critical phenomena, Vol. 1. London, New York: Academic Press 1972 
7. Galavotti, G., Martin-Lof, A., Miracle-Sole, S.: Some problems connected with the description of coexisting phases at low temperatures in the Ising model. In Lecture Notes in Physics, Vol.20. Berlin, Heidelberg, New York: Springer 1973

8. Pirogov, S.A., Sinai, Ya.G.: Phase diagrams of classical lattice systems, I, II. Theor. Math. Phys. 25, 1185-1192 (1975) and 26, 39-49 (1976)

9. Gruber, C., Hintermann, A., Merlini, D.: Group analysis of classical lattice systems. Lecture Notes in Physics, Vol.60. Berlin, Heidelberg, New York: Springer 1977

10. Wu, F.Y.: The Potts model. Rev. Mod. Phys. 54 (1), 235-268 (1982)

11. Bricmont, J., Kuroda, K., Lebowitz, J.L.: Surface tension and phase coexistence for general lattice systems. J. Stat. Phys. 33, 59-75 (1983)

12. Baxter, R.J.: Magnetization discontinuity of the two dimensional Potts model. J. Phys. A 15, 3329-3340 (1982)

13. Bricmont, J., Lebowitz, J.L., P fister, C.-E.: On the surface tension of lattice systems. Ann. N.Y. Acad. Sci. 337, 214 (1980)

14. Pfister, C.-E.: Translation invariant equilibrium states of ferromagnetic abelian systems. Commun. Math. Phys. 86, 375-390 (1982)

15. Hintermann, A., Kunz, H., Wu, F.Y.: Exact results for the Potts model in two dimensions. J. Stat. Phys. 19, 623-632 (1978)

16. Griffith, P., Harris, J.: Principles of algebric geometry. New York: Wiley 1956

17. Alexandroff, P.: Combinatorial topology. Vols. 1-3. Rochester, New York: Graylock 1956

18. Ginibre, J.: General formulation of Griffiths inequalities. Commun. Math. Phys. 16, 310-328 (1970)

19. Messager, A., Miracle-Sole, S., Pfister, C.-E.: Correlation inequalities and uniqueness of the equilibrium states in the plane rotator ferromagnetic model. Commun. Math. Phys. 59, 19-29 (1978)

20. Ruelle, D.: Statistical mechanics rigourous results. New York, Amsterdam: Benjamin 1969

21. Fröhlich, J., Israel, R., Lieb, E., Simon, B.: Phase transitions and reflection positivity. I, II. Commun. Math. Phys. 62, 1-37 (1978) and J. Stat. Phys. 22, 297-347 (1980)

22. Dobrushin, R.L., Shlosman, S.B.: Phases corresponding to the local energy minima

23. Laanait, L.,Messager, A., Ruiz, J.: Discontinuity of the string tension in the four dimensional pure gauge Potts models (in preparation)

24. Dobrushin, R.L.: Existence of phase transitions in two and three dimensional lattice model. Teor. Veroyatn. Primen. 10, 209-230 (1965)

25. Drouffe, J.M.: Transitions and duality in gauge lattice systems. Phys. Rev. D 18, 1174-1182 (1978)

26. Zinovev, Y.M.: Duality in abelian gauge theories on a lattice. Theor. Math. Phys. 43, 481-487 (1980)

27. Druhl, K., Wagner, H.: Algebraic formulation of duality transformation for abelian lattice models. Ann. Phys. 141, 225-253 (1982)

28. Dobrushin, R.L., Pechersky, E.A.: Colloquium on random fields (Esztergom, June 1979)

29. Weng, Chi-Yuan, Griffiths, R.B., Fisher, M.: Critical temperatures of anisotropic Ising lattices. I. Lower bounds. Phys. Rev. 162, 475 (1967)

30. Martirosian, D.H.: Translation invariant Gibbs states in the $q$ states Potts model. Commun. Math. Phys. (in press)

Communicated by Ya. G. Sinai

Received November 15, 1984; in revised form August 20, 1985 
